\title{
Antimicrobial Use Guidelines for Treatment of Urinary Tract Disease in Dogs and Cats: Antimicrobial Guidelines Working Group of the International Society for Companion Animal Infectious Diseases
}

\author{
J. Scott Weese, ${ }^{1}$ Joseph M. Blondeau, ${ }^{2}$ Dawn Boothe, ${ }^{3}$ Edward B. Breitschwerdt, ${ }^{4}$ \\ Luca Guardabassi, ${ }^{5}$ Andrew Hillier, ${ }^{6}$ David H. Lloyd, ${ }^{7}$ Mark G. Papich, ${ }^{4}$ Shelley C. Rankin, ${ }^{8}$ \\ John D. Turnidge, ${ }^{9,10}$ and Jane E. Sykes ${ }^{11}$ \\ ${ }^{1}$ Department of Pathobiology, Ontario Veterinary College, University of Guelph, Guelph, ON, Canada N1G 2W1 \\ ${ }^{2}$ Clinical Microbiology, Royal University Hospital and University of Saskatchewan, Saskatoon, SK, Canada S7N 0W8 \\ ${ }^{3}$ College of Veterinary Medicine, Auburn University, Auburn, AL 36849, USA \\ ${ }^{4}$ College of Veterinary Medicine, North Carolina State University, Raleigh, NC 27606, USA \\ ${ }^{5}$ Department of Veterinary Disease Biology, Faculty of Life Sciences, University of Copenhagen, \\ 1870 Frederiksberg C Copenhagen, Denmark \\ ${ }^{6}$ College of Veterinary Medicine, The Ohio State University, Columbus, OH 43210, USA \\ 7 Department of Clinical Sciences, Royal Veterinary College, North Mymms, Hertfordshire AL9 7TA, UK \\ ${ }^{8}$ School of Veterinary Medicine, University of Pennsylvania, Philadelphia, PA 19104, USA \\ ${ }^{9}$ Division of Laboratory Medicine, Women's and Children's Hospital, North Adelaide, SA 5006, Australia \\ ${ }^{10}$ University of Adelaide, Adelaide, SA 5000, USA \\ ${ }^{11}$ Department of Medicine and Epidemiology, University of California, Davis, Davis, CA 95616, USA
}

Correspondence should be addressed to J. Scott Weese, jsweese@uoguelph.ca

Received 16 February 2011; Accepted 31 March 2011

Academic Editor: Remo G. Lobetti

Copyright () $2011 \mathrm{~J}$. Scott Weese et al. This is an open access article distributed under the Creative Commons Attribution License, which permits unrestricted use, distribution, and reproduction in any medium, provided the original work is properly cited.

Urinary tract disease is a common reason for use (and likely misuse, improper use, and overuse) of antimicrobials in dogs and cats. There is a lack of comprehensive treatment guidelines such as those that are available for human medicine. Accordingly, guidelines for diagnosis and management of urinary tract infections were created by a Working Group of the International Society for Companion Animal Infectious Diseases. While objective data are currently limited, these guidelines provide information to assist in the diagnosis and management of upper and lower urinary tract infections in dogs and cats.

\section{Introduction}

Urinary tract disease is commonly encountered in dogs and cats and accounts for significant use (and presumably also overuse and misuse) of antimicrobials. Improper therapy can lead to a variety of patient health (e.g., failure to resolve infection), economic (e.g., need for repeated or prolonged treatment), public health (e.g., antimicrobial resistance) and regulatory (e.g., antimicrobial use) concerns.
In human medicine, antimicrobial use guidelines such as those developed by the Infectious Diseases Society of America (IDSA) are widely respected and provide excellent guidance to physicians on management of various infectious diseases, including urinary tract infections (UTIs) $[1,2]$. Such guidelines can be directly used or form the basis of hospital-level antimicrobial use guidelines. The impact of national or international guidelines is difficult to assess, but implementation of antimicrobial use guidelines at the 
hospital level has been shown to significantly improve antimicrobial prescribing practices, either alone or as part of a broader antimicrobial stewardship program [3-5].

This document contains guidelines developed in 2010 by the Antimicrobial Guidelines Working Group of the International Society for Companion Animal Infectious Diseases. During the course of guideline development, it became abundantly clear that there are significant limitations in objective, published information. Accordingly, recommendations are based on available data, whenever present, along with expert opinion, considering principles of infectious diseases, antimicrobial therapy, antimicrobial resistance, pharmacology, and internal medicine. Corresponding guidelines for human medicine were evaluated, with careful consideration of the abundant differences between species.

As with all guidelines, these should be interpreted as general recommendations that are reasonable and appropriate for the majority of cases. The Working Group acknowledges the variability between cases, and these guidelines should not be considered as standards of care that must be followed in all circumstances. Rather, they should be considered the basis of decision-making, with the potential that different or additional approaches may be required in a minority of cases. Further, while these guidelines are designed as international guidelines, appropriate for all jurisdictions, the Working Group realizes that regional differences (e.g., antimicrobial resistance rates, antimicrobial availability, prescribing regulations) exist.

\section{Simple Uncomplicated Urinary Tract Infection}

Simple uncomplicated UTI is a sporadic bacterial infection of the bladder in an otherwise healthy individual with normal urinary tract anatomy and function. The presence of relevant comorbidities (e.g., diabetes mellitus, urinary or reproductive tract conformational abnormalities) or 3 or more episodes per year indicates complicated or recurrent UTI, respectively, as are discussed below.

Clinically significant infection implies the presence of a clinical abnormality and is characterized by dysuria, pollakiuria, and/or increased urgency of urination along with the presence of bacteria in urine [2]. These clinical signs are not pathognomonic for infection and can also be caused by noninfectious conditions. Additionally, bacteria can be present in the urine in the absence of clinical signs (covert bacteriuria/subclinical bacteriuria). Therefore, the clinician must interpret the clinical evaluation, gross and cytological appearance of the urine, and bacterial culture results in parallel to determine the likelihood of a clinically significant UTI.

\subsection{Summary of Recommendations for Uncomplicated UTIs}

2.1.1. Diagnosis of Uncomplicated UTIs. Proper and timely diagnosis is critical for management of UTIs. Proper diagnosis allows for determination of both the need for antimicrobials and optimal drugs.
As discussed above, clinical signs are nonspecific and should not be used alone for diagnosis of UTI. Rather, the presence of clinical abnormalities should indicate the need for further testing.

Sediment analysis alone is inadequate for diagnosis of UTIs because of problems regarding the variable quality of interpretation, stain contamination, and false positive results from bacteriuria in the absence of clinical infection. Hematuria and proteinuria are often present with a UTI, but they are nonspecific and may be caused by noninfectious conditions. The presence of pyuria and bacteriuria does, however, provide supporting evidence of a UTI. Sediment analysis is a useful adjunctive measure to consider in conjunction with clinical signs and culture results. All individuals performing analysis should be proficient at the technique, ideally through formal training and ongoing education and quality control.

Complete urinalysis, including urine-specific gravity, urine glucose level determination, and examination of the sediment for crystalluria is considered a minimum database for evaluation of suspected UTI and may be helpful to investigate underlying causes of infection, if present.

Aerobic bacterial culture and susceptibility testing should be performed in all cases, to confirm the presence of infection, identify the presence of resistant bacteria that may not respond to initial therapy, to help differentiate reinfection from relapse should a UTI return, and to provide the clinician with guidance regarding the most common bacteria causing UTI in their practice and local susceptibility patterns.

Cystocentesis should be used for sample collection. Catheterized samples can be evaluated for culture but cystocentesis samples are preferred. Free-catch (midstream voiding or manual expression) samples should not be used. It is imperative that a quantitative culture be performed.

Urine samples for culture and susceptibility testing should be refrigerated immediately after collection and submitted to the laboratory as quickly as possible. Results of samples that take 24 hours or more to reach the laboratory should be interpreted with caution because of the potential for both false positive and false negative results, particularly if a urine preservative was not used. Testing of refrigerated samples greater than 24 hours old is acceptable if samples contain a urine preservative; otherwise, retesting is recommended.

The use of specific urine transportation tubes is recommended, provided they are appropriate for the volume of urine that is collected. The use of new or alternative techniques or materials intended to facilitate successful culture such as inoculation of "urine paddles" in clinics is a reasonable alternative to traditional sample collection approach to try to optimize bacterial recovery. The preferred approach is for paddles to be inoculated in clinics and promptly submitted to a diagnostic laboratory for incubation and subsequent testing.

The use of traditional culture methods in clinics may be a reasonable alternative to submission to outside laboratories. In-clinic testing can minimize the impact of sample deterioration that occurs between sample collection in the clinic 
and processing at the laboratory and can be cost-effective for screening purposes. However, bacterial isolation should only be attempted in clinics with appropriate laboratory facilities, proper equipment, proper biosafety level 2 (BSL-2) containment and waste management, and adequately trained individuals. Quantitative culture should be performed. Incubation of urine paddles in the clinic can be performed, but this approach has the same biosafety requirements as incubation of culture plates.

Identification and susceptibility testing must only be performed if there is adequate biocontainment and properly trained staff. Only protocols that include reference strains for quality control testing and have been standardized by an appropriate organization (i.e., Clinical and Laboratory Standards Institute (CLSI), European Union Committee on Antimicrobial Susceptibility Testing (EUCAST), or equivalent) should be followed.

If culture is being performed to screen samples and send isolates (on plates, paddles, swabs, or any other approach) to a diagnostic laboratory for subsequent testing, clinicians must contact their laboratory to determine if those items will be accepted. In all cases in which isolates are shipped, regional regulations must be followed regarding shipment of bacteria. If a veterinary clinic is unable to satisfy BSL2 containment and properly ship isolates, the use of these techniques is discouraged.

The advantage of quantitative culture techniques lies in the availability to determine the level of bacterial growth (colony counts), which can be used in interpreting the relevance of results. For samples collected by cystocentesis, any level of bacterial growth may be significant, although samples from a UTI typically contain $\geq 10^{3}$ colony forming units $(\mathrm{CFU}) / \mathrm{mL}[6]$. The colony count and the identity of the organism isolated should be considered in all situations. Small numbers of minimally pathogenic skin commensals (i.e., coagulase negative staphylococci) likely represent contamination.

For samples collected via catheter, bacterial counts $\geq 10^{4} \mathrm{CFU} / \mathrm{mL}$ in males and $\geq 10^{5}$ in females are typically considered significant. Samples with lower counts should be interpreted with caution and ideally repeated prior to treatment to confirm the same organism can be demonstrated. Samples obtained in male dogs by catheterization are usually adequate so long as proper sterile technique was performed to obtain the sample. Positive cultures obtained from catheterized female dogs should be confirmed with a cystocentesis unless medically contraindicated.

Although it has been suggested that bacterial counts of greater than or equal to $10^{5} \mathrm{CFU} / \mathrm{mL}$ in dogs and $10^{4} \mathrm{CFU} / \mathrm{mL}$ in cats are significant for free-catch samples [6], the potential for high-level contamination is present and therefore results from free-catch samples are not considered diagnostic. Cystocentesis should be performed to confirm positive culture results from free-catch samples, unless medically contraindicated.

Susceptibility testing should be performed according to accepted standards, such as those published by the CLSI or EUCAST, or another internationally recognized public standard. The interpretive criteria for susceptibility testing and breakpoints for systemic infections also apply to UTIs. Those drugs for which urinary tract-specific breakpoints have been provided are limited to ampicillin or amoxicillin in $\operatorname{dogs}(\leq 8 \mu \mathrm{g} / \mathrm{mL})$, amoxicillin-clavulanate in dogs and cats $<8 / 4 \mu \mathrm{g} / \mathrm{mL})$, and nitrofurantoin.

\subsubsection{Treatment of Uncomplicated UTIs. Antimicrobial ther-} apy is indicated in most cases while awaiting culture and susceptibility results to relieve patient discomfort. In most situations, initial therapy should consist of amoxicillin (11$15 \mathrm{mg} / \mathrm{kg}$ PO q8h) or trimethoprim-sulfonamide $(15 \mathrm{mg} / \mathrm{kg}$ PO q12h) (Tables 1 and 2). Amoxicillin/clavulanic acid $(12.5-25 \mathrm{mg} / \mathrm{kg} \mathrm{PO} \mathrm{q} 8 \mathrm{~h}$ ) is an acceptable option but is not recommended initially because of the lack of evidence regarding the need for clavulanic acid and the desire to use the narrowest spectrum that is possible while maintaining optimal efficacy.

Veterinarians should be aware of the pathogen and antimicrobial resistance trends among urinary pathogens isolated from patients in their clinic. Reports of resistance rates from other institutions or broader surveillance programs can provide some information but it is not a replacement for clinic-level information.

Changes in local resistance patterns for urinary pathogens should be monitored. As baseline resistance rates of the most common organisms to a first-line drug increase, consideration should be given to changing the empirical drug choice. Objective data regarding the prevalence of resistance that indicates a need to change initial therapy is lacking; however the Working Group considers 10\% to be a reasonable standard. Accordingly, if baseline resistance rates to a given drug from non biased sample collection exceed $10 \%$, the drug chosen for initial therapy should be changed to another of the recommended initial choices. Care should be taken when determining rates. Decisions should be based on cultures from an appropriate patient population (i.e., animals presented for simple uncomplicated UTI). If culture data are biased towards animals with refractory or recurrent infections, then the baseline prevalence of resistance in the uncomplicated UTI population could be overestimated. Therefore, determination of this prevalence should involve a comparable population of primary uncomplicated UTIs and should involve the use of proper breakpoints.

If culture and susceptibility testing indicates the presence of an isolate that is resistant in vitro to initial therapy but there has been apparent clinical response, maintaining the current treatment is acceptable provided a follow-up urinalysis, including culture, is performed after treatment has been completed to ensure resolution of infection.

If culture and susceptibility data indicate that the isolate is not susceptible to the chosen antimicrobial and there is a lack of clinical response, then therapy with the original drug should be discontinued and treatment with an alternative drug begun. Factors that should be considered when choosing the antimicrobial include the susceptibility of the bacterium, potential adverse effects, and issues regarding prudent use of certain antimicrobials and antimicrobial classes. More detailed discussion regarding drug selection is provided below under Complicated UTI. 
TABLE 1: Summary of first-line antimicrobial options for UTIs in the dog and cat.

\begin{tabular}{ll}
\hline Infection Type & First-line drug options \\
\hline Uncomplicated UTI & $\begin{array}{l}\text { Amoxicillin, trimethoprim-sulfonamide } \\
\text { Guided by culture and susceptibility testing, but consider amoxicillin or trimethoprim-sulfonamide initially }\end{array}$ \\
Subclinical bacteriuria & $\begin{array}{l}\text { Antimicrobial therapy not recommended unless high risk for ascending infection. If so, treat as per } \\
\text { complicated UTI }\end{array}$ \\
Pyelonephritis & Start with a fluoroquinolone, with re-assessment based on culture and susceptibility testing \\
\hline
\end{tabular}

Adequate evidence regarding duration of treatment is lacking, precluding the ability to make a specific recommendation for treatment duration. Typically, uncomplicated UTIs are treated for 7-14 days. However, the Working Group acknowledges the likelihood that a shorter treatment time ( $\leq 7$ days) may be effective. Accordingly, in the absence of objective data, 7 days of appropriate antimicrobial treatment is reasonable. Clinical trials supporting shorter durations for treatment of UTIs in dogs and cats are strongly encouraged.

There is currently insufficient evidence supporting the use of other adjunctive treatment measures (e.g., cranberry extract) for uncomplicated UTIs in animals.

2.1.3. Monitoring Response to Treatment of Uncomplicated UTIs. There is no indication for measures beyond monitoring of clinical signs. Provided the full course of antimicrobials is administered correctly, there is no evidence that intraor posttreatment urinalysis or urine culture is indicated in the absence of ongoing clinical signs of UTI.

\section{Complicated Urinary Tract Infection}

A complicated UTI is an infection that occurs in the presence of an anatomic or functional abnormality or a comorbidity that predisposes the patient to persistent infection, recurrent infection, or treatment failure [7]. In humans, the concurrent presence of prostatitis, urinary calculi, a neurogenic bladder, pregnancy, diabetes mellitus, or immunocompromising disorders also defines a complicated UTI [7], and it is reasonable to apply this to companion animals. An identifiable abnormality is not always present, because of the difficulty diagnosing some anatomical, functional, metabolic, or other abnormalities. Recurrent UTIs, as defined by the presence of 3 or more episodes of UTI during a 12-month period [8], also indicate complicated infection.

Recurrent UTIs can more loosely be defined as reinfection or relapse. While definitive determination of relapse versus reinfection is difficult (or often impossible), consideration of whether reinfection or relapse is most likely is important.

Reinfection is recurrence of a UTI within 6 months of cessation of previous, apparently successful treatment and isolation of a different microorganism. If the same bacterial species is present, genotyping is ideal to determine whether the same strain is present; however this is uncommonly available. Evaluation of antimicrobial susceptibility pattern can be helpful, although unrelated organisms can have the same susceptibility pattern and changes in susceptibility can occur in individual strains. Therefore, evaluation of susceptibility pattern is useful but not definitive.

Relapse is recurrence of a UTI within 6 months of cessation of previous, apparently successful treatment and isolation of an indistinguishable organism from the one that was present previously, which is presumably because of failure to completely eliminate the pathogen. In general, relapses tend to occur earlier than reinfections (i.e., within weeks rather than months) and are characterized by a period of apparent bladder sterility during treatment. It should be recognized that isolation of the same bacterial species, even if the same antimicrobial susceptibility pattern is present, does not definitively indicate that the organism was not successfully eliminated, since reinfection with the same strain or a phenotypically (or even genotypically) indistinguishable strain cannot be ruled out.

A refractory infection is similar to a relapse except that it is characterized by persistently positive results using culture during treatment (despite in vitro susceptibility to the antimicrobial), with no period of eliminated of bacteriuria during or after treatment.

\subsection{Summary of Recommendations for Complicated UTIs}

3.1.1. Diagnosis of Complicated UTIs. General principles of diagnosis, as discussed for simple uncomplicated UTI, apply for complicated UTIs. A diagnosis of recurrent UTI should never be based on clinical signs or urine sediment examination alone. Bacterial culture and susceptibility testing should be performed in all instances to confirm recurrent UTI.

Efforts must be made to determine any underlying factors that could be associated with recurrence or relapse. A complete blood cell count, serum biochemical profile, urinalysis, imaging and, if deemed appropriate, endocrine testing should be performed. A complete physical examination, including rectal palpation and examination of the vulva, is required. Referral to a specialist in internal medicine or surgery (particularly in females) should be considered to further investigate underlying causes (e.g., cystoscopy). If an underlying cause cannot be found and corrected, it is possible therapy will ultimately be unsuccessful.

Client compliance with previous antimicrobial treatment should be investigated. This is particularly important in cases where relapse is suspected.

If cystotomy is being performed, culture of a bladder wall biopsy as well as any uroliths that might be present is recommended [9]. 
TABLE 2: Antimicrobial treatment options for urinary tract infections in the dog and cat.

\begin{tabular}{|c|c|c|}
\hline Drug & Dose & Comments \\
\hline Amoxicillin & $11-15 \mathrm{mg} / \mathrm{kg} \mathrm{PO} \mathrm{q} 8 \mathrm{~h}$ & $\begin{array}{l}\text { Good first-line option for UTIs. Excreted in urine } \\
\text { predominantly in active form if normal renal function is } \\
\text { present. Ineffective against beta-lactamase-producing bacteria. }\end{array}$ \\
\hline Amikacin & $\begin{array}{l}\text { Dogs: } 15-30 \mathrm{mg} / \mathrm{kg} \text { IV/IM/SC q24h } \\
\text { Cats: } 10-14 \mathrm{mg} / \mathrm{kg} \text { IV/IM/SC q24h }\end{array}$ & $\begin{array}{l}\text { Not recommended for routine use but may be useful for } \\
\text { treatment of multidrug resistant organisms. Potentially } \\
\text { nephrotoxic. Avoid in animals with renal insufficiency. }\end{array}$ \\
\hline Amoxicillin/clavulanate & $\begin{array}{l}12.5-25 \mathrm{mg} / \mathrm{kg} \text { PO q } 8 \mathrm{~h}(\text { dose based on } \\
\text { combination of amoxicillin }+ \\
\text { clavulanate) }\end{array}$ & $\begin{array}{l}\text { Not established whether there is any advantage over amoxicillin } \\
\text { alone. }\end{array}$ \\
\hline Ampicillin & & $\begin{array}{l}\text { Not recommended because of poor oral bioavailability. } \\
\text { Amoxicillin is preferred. }\end{array}$ \\
\hline Cephalexin, Cefadroxil & $12-25 \mathrm{mg} / \mathrm{kg} \mathrm{PO}$ q12h & $\begin{array}{l}\text { Enterococci are resistant. Resistance may be common in } \\
\text { Enterobacteriaceae in some regions. }\end{array}$ \\
\hline Cefovecin & $\begin{array}{l}8 \mathrm{mg} / \mathrm{kg} \text { single SC injection. Can be } \\
\text { repeated once after } 7-14 \text { days. }\end{array}$ & $\begin{array}{l}\text { Should only be used in situations where oral treatment is } \\
\text { problematic. Enterococci are resistant. Pharmacokinetic data } \\
\text { are available to support the use in dogs and cats, with a duration } \\
\text { of } 14 \text { days (dogs) and } 21 \text { days (cats). The long duration of } \\
\text { excretion in the urine makes it difficult to interpret } \\
\text { posttreatment culture results. }\end{array}$ \\
\hline Cefpodoxime proxetil & 5 to $10 \mathrm{mg} / \mathrm{kg}$ q24h PO & Enterococci are resistant. \\
\hline Ceftiofur & $2 \mathrm{mg} / \mathrm{kg}$ q12-24h SC & $\begin{array}{l}\text { Approved for treatment of UTIs in dogs in some regions. } \\
\text { Enterococci are resistant. }\end{array}$ \\
\hline Chloramphenicol & $\begin{array}{l}\text { Dogs: } 40-50 \mathrm{mg} / \mathrm{kg} \text { PO q } 8 \mathrm{~h} \\
\text { Cats: } 12.5-20 \mathrm{mg} / \mathrm{kg} \text { PO q12h }\end{array}$ & $\begin{array}{l}\text { Reserved for multidrug resistant infections with few other } \\
\text { options. Myelosuppression can occur, particularly with } \\
\text { long-term therapy. Avoid contact by humans because of rare } \\
\text { idiosyncratic aplastic anemia. }\end{array}$ \\
\hline Ciprofloxacin & $30 \mathrm{mg} / \mathrm{kg}$ PO q24h & $\begin{array}{l}\text { Sometimes used because of lower cost than enrofloxacin. Lower } \\
\text { and more variable oral bioavailability than enrofloxacin, } \\
\text { marbofloxacin, and orbifloxacin. Difficult to justify over } \\
\text { approved fluoroquinolones. Dosing recommendations are } \\
\text { empirical. }\end{array}$ \\
\hline Doxycycline & $3-5 \mathrm{mg} / \mathrm{kg}$ PO q12h & $\begin{array}{l}\text { Highly metabolized and excreted through intestinal tract, so } \\
\text { urine levels may be low. Not recommended for routine uses. }\end{array}$ \\
\hline Enrofloxacin & $\begin{array}{l}5 \mathrm{mg} / \mathrm{kg} \text { PO q24h (cats) } \\
10-20 \mathrm{mg} / \mathrm{kg} \text { q24h (dogs) }\end{array}$ & $\begin{array}{l}\text { Excreted in urine predominantly in active form. Reserve for } \\
\text { documented resistant UTIs but good First-line choice for } \\
\text { pyelonephritis ( } 20 \mathrm{mg} / \mathrm{kg} \text { PO q24h). Limited efficacy against } \\
\text { enterococci. Associated with risk of retinopathy in cats. Do not } \\
\text { exceed } 5 \mathrm{mg} / \mathrm{kg} / \mathrm{d} \text { of enrofloxacin in cats. }\end{array}$ \\
\hline Imipenem-cilastatin & $5 \mathrm{mg} / \mathrm{kg}$ IV/IM q6-8h & $\begin{array}{l}\text { Reserve for treatment of multidrug-resistant infections, } \\
\text { particularly those caused by Enterobacteriaceae or Pseudomonas } \\
\text { aeruginosa. Recommend consultation with a urinary or } \\
\text { infectious disease veterinary specialist or veterinary } \\
\text { pharmacologist prior to use. }\end{array}$ \\
\hline Marbofloxacin & $2.7-5.5 \mathrm{mg} / \mathrm{kg}$ PO q24h & $\begin{array}{l}\text { Excreted in urine predominantly in active form. Reserve for } \\
\text { documented resistant UTIs but good First-line choice for } \\
\text { pyelonephritis. Limited efficacy against enterococci. }\end{array}$ \\
\hline Meropenem & $8.5 \mathrm{mg} / \mathrm{kg} \mathrm{SC} / \mathrm{IV}$ q $12(\mathrm{SC})$ or 8 (IV)h & $\begin{array}{l}\text { Reserve for treatment of multidrug-resistant infections, } \\
\text { particularly those caused by Enterobacteriaceae or Pseudomonas } \\
\text { aeruginosa. Recommend consultation with a urinary or } \\
\text { infectious disease veterinary specialist or veterinary } \\
\text { pharmacologist prior to use. }\end{array}$ \\
\hline Nitrofurantoin & $4.4-5 \mathrm{mg} / \mathrm{kg} \mathrm{PO} \mathrm{q} 8 \mathrm{~h}$ & $\begin{array}{l}\text { Good second-line option for simple uncomplicated UTI, } \\
\text { particularly when multidrug-resistant pathogens are involved. }\end{array}$ \\
\hline Orbifloxacin & $\begin{array}{l}\text { Tablets: } 2.5-7.5 \mathrm{mg} / \mathrm{kg} \text { PO q24h; oral } \\
\text { suspension: } 7.5 \mathrm{mg} / \mathrm{kg} \text { PO q24h (cats) or } \\
2.5-7.5 \mathrm{mg} / \mathrm{kg} \text { PO q24h (dogs) }\end{array}$ & Excreted in urine predominantly in active form. \\
\hline
\end{tabular}


TABLE 2: Continued.

\begin{tabular}{lll}
\hline Drug & Dose & \multicolumn{1}{c}{ Comments } \\
\hline Trimethoprim-sulfadiazine & $\begin{array}{l}\text { Note: dosing is based on total } \\
\text { trimethoprim + sulfadiazine } \\
\text { concentration }\end{array}$ & $\begin{array}{l}\text { Good first-line option. Concerns regarding idiosyncratic and } \\
\text { immune-mediated adverse effects in some patients, especially } \\
\text { with prolonged therapy. If prolonged }(>7 \mathrm{~d}) \text { therapy is } \\
\text { anticipated, baseline Schirmer's tear testing is recommended, } \\
\text { with periodic re-evaluation and owner monitoring for ocular } \\
\text { discharge. Avoid in dogs that may be sensitive to potential } \\
\text { adverse effects such as KCS, hepatopathy, hypersensitivity, and } \\
\text { skin eruptions. }\end{array}$ \\
\hline
\end{tabular}

3.1.2. Treatment of Complicated UTIs. If the clinical condition of the patient permits, consideration should be given to waiting for culture results before starting therapy. If treatment must be initiated immediately for patient care reasons, a drug should be selected from those recommended for initial treatment of simple uncomplicated UTI. Where possible, the drug class used should be different from that used to treat the prior UTI(s) (i.e., if amoxicillin was used initially, start treatment with trimethoprim-sulfadiazine).

After treatment has been initiated, continued treatment should be based on the results of culture and susceptibility testing. Preference should be given to drugs that are excreted in urine predominantly in an active form. Classes of drugs that are not predominantly excreted in urine in active form (e.g., macrolides) should be avoided. Referral or consultation with specialist to determine the appropriate approach is reasonable.

The susceptibility test will typically use the S/I/R classification (Susceptible, Intermediate, and Resistant). If resistant, treatment is likely to fail and the drug should not be prescribed. If susceptible, there is a greater likelihood of clinical success (resolution of clinical signs of UTI) using normal treatment regimens; however factors such as drug absorption, drug excretion, drug inactivation, biofilm, necrotic debris, the presence of foreign materials, development of drug resistance during treatment, inducible resistance, laboratory error, and various comorbidities can impact on the success of an individual treatment. The use of drugs reported as intermediate is appropriate in situations when the drug is physiologically concentrated at the target site or if the dosage can be increased.

If treatment was initiated before culture, susceptibility results were available, and the isolated organism is resistant to the antimicrobial that was initially chosen, a change to a more active drug based on susceptibility testing should be made.

If more than one bacterial species is identified on initial culture, the relevance of the each organism should be considered, based on the bacterial counts and the pathogenicity of the organisms. For example, when present in a mixed infection, anecdotal evidence suggests that infection by Enterococcus spp. will often resolve when the other organism is successfully treated. Ideally, antimicrobial therapy should be directed against both organisms. In some instances, an antimicrobial effective against both organisms will not be available. Combination therapy that would be potentially effective against both organisms should be considered. In some situations, a reasonable drug or drug combination may not be available. Targeting therapy towards the organism perceived as the most clinically relevant is a reasonable approach, provided there is no evidence of pyelonephritis or underlying disease that increases the risk of systemic or ascending infection with an organism otherwise of limited primary pathogenicity.

There is no supporting evidence for administration of other drugs (e.g., clarithromycin) for the purpose of breaking down bacterial biofilm.

There is no supporting evidence that direct instillation of antimicrobials, antiseptics, or DMSO directly into the bladder via a urinary catheter is effective for treatment of recurrent UTIs. These compounds are quickly flushed out of the bladder when the animal urinates and may be locally irritating.

Any underlying causes should be managed appropriately, whenever possible.

Evidence supporting the duration of therapy for complicated UTI does not exist. Typically, 4 weeks of treatment has been recommended. The Working Group agrees that it is likely that shorter courses of appropriate treatment might be effective in some or all situations. In the face of insufficient data supporting a shorter course of therapy, 4 weeks of treatment is a reasonable recommendation. In animals with a nonrecurrent but complicated UTI (e.g., diabetic animal with a first instance of UTI and whose infection would have been classified as uncomplicated if the comorbidity was not present), shorter-term therapy may be more reasonable.

3.1.3. Monitoring Response to Treatment. Urine culture should be considered 5-7 days after initiation of therapy, particularly in patients with a history of relapsing or refractory infection, or those considered at high risk for ascending or systemic infection.

Any bacterial growth during treatment indicates potential treatment failure and should prompt immediate reevaluation. Referral or consultation with a specialist is recommended.

Urine culture is recommended 7 days after cessation of therapy in all cases. (If the patient was treated with cefovecin, the prolonged excretion of the drug must be considered. Optimal timing of sampling in such cases is unclear, but testing 3 weeks after the last dose may be reasonable.) If a positive urine culture is obtained after treatment, more in-depth investigation of predisposing factors for relapse or reinfection should be performed. Referral should be strongly 
considered. Unless there is clear evidence for the reason for failure, retreatment without any other investigation is not recommended. If no signs of disease are present, these cases should be managed as described for subclinical bacteriuria.

If there is lack of clinical response to treatment or if clinical signs of UTI recur after apparently successful treatment, the animal should be managed again as described above, with particular emphasis on determination of underlying causes. Referral is strongly recommended.

3.1.4. Preventive Measures. There is insufficient evidence to recommend use of either pulse (intermittent) or chronic lowdose therapy for prevention of UTIs. The effect of these practices on emergence of resistance should be considered. There is anecdotal evidence that a small percentage of animals with severe clinical signs may require such an approach, but only after thorough evaluation for underlying causes, ideally by a specialist in small animal internal medicine. Although there may be some theoretical benefit for the administration of urinary antiseptics such as methenamine (methenamine hippurate), there is currently insufficient evidence available to assess the effectiveness of these treatments in animals.

There is currently insufficient evidence to support the administration of other adjunctive therapies to prevent recurrent infections. Although there is some evidence for a benefit of nutritional supplements (e.g., cranberry juice extract) for this indication in humans, the evidence is not strong and some studies have shown no effect [10-13]. Currently, there are no data to support the benefits of these measures in veterinary medicine. When effective in people, dietary supplements may help to prevent but not treat UTIs. When selecting cranberry-based treatments, one should be aware of the variability in quality and potency of over-the-counter products and, based on human studies and in vitro work, should choose an extract that has a higher concentration of proanthocyanidins, the antioxidants thought to be responsible for antibacterial effects.

\section{Subclinical Bacteriuria}

Subclinical bacteriuria is the presence of bacteria in the urine as determined by positive bacterial culture, in the absence of clinical and cytological evidence of UTI.

4.1. Summary of Recommendations. Treatment may not be necessary in animals that have no clinical signs of UTI and no evidence of UTI based on examination of urine sediment.

In some circumstances, treatment may be considered if there is concern that there is a particularly high risk of ascending or systemic infection (e.g., immunocompromised patients, patients with underlying renal disease) or that the bladder may be a focus of extraurinary infection. Diagnosis and management of the underlying cause is critical and treatment should not be used as a replacement for proper diagnosis and management.

The presence of multidrug-resistant bacterium does not represent, by itself, an indication for treatment. Anecdotal information suggests that multidrug-resistant organisms will sometimes be replaced with susceptible organisms if treatment is withheld, and then treatment with routine antimicrobials may be more practical if decolonization is desired or if clinical disease develops.

\section{Urinary Catheters}

UTI and subclinical bacterial colonization of the bladder are commonly identified in dogs with indwelling urinary catheters $[14,15]$. Differentiating these two is important because the approach to management of infection versus colonization is different.

5.1. Animals with Urinary Catheters: No Clinical Signs of Infection. Culture for diagnosis of the presence of bacteria in urine is not recommended in the absence of clinical signs consistent with an active infection.

It is not necessary to treat catheterized animals with bacteriuria in the absence of clinical or cytological evidence supporting the presence of an infection.

Prophylactic antimicrobial therapy for prevention of UTI in catheterized animals is never indicated.

5.2. Animals with Urinary Catheters: When Urinary Catheter Is Being Removed. There is no evidence supporting the need to culture the catheter tip at time of catheter removal since catheter tip culture results are not predictive of development of catheter-associated UTI [16]. There is also no evidence supporting routine culture of urine after catheter removal. However, culture of the urine (ideally collected by cystocentesis after catheter removal) may be reasonable in patients in which the risk and implications of UTI are high (e.g., recently obstructed male cat with at high risk for reobstruction). Clinical monitoring and cytological examination to detect a potential UTI are preferred to urine culture in a patient with no signs of UTI.

There is no indication for routine (prophylactic) antimicrobial treatment following urinary catheter removal in an animal with no clinical or cytological evidence of active UTI.

\section{Animals with a Urinary Catheter and Signs of a UTI}

UTI should be suspected in catheterized animals exhibiting clinical signs of infection. However, such patients may not be easily identified. As such, infection should be suspected in all cases of fever of unknown origin or bacteremia with an unknown focus. Infection should also be suspected when there are gross or cytological (i.e., hematuria, pyuria) abnormalities.

Urine culture should always be performed if infection is suspected. If a catheter must remain in place and signs of UTI are present, the catheter should be replaced and a urine sample collected through the new catheter for culture. Several milliliters of urine should be withdrawn to clear the catheter and discarded prior to obtaining the sample for culture.

When possible, catheters should be removed and urine sampled by cystocentesis after an appropriate period of time has passed for the bladder to fill with urine. Less ideal is a 
sample collected from the urinary catheter prior to removal, in which case positive cultures should ideally be confirmed with a subsequent cystocentesis sample. In all instances, cultures should be quantitative.

Urine culture should never be performed from the collection bag.

Culture of the catheter tip after removal is not recommended.

Treatment is more likely to be successful if the catheter can be removed. The costbenefit of removing or retaining the catheter should be considered in the context of management of the UTI and the patient's underlying disease condition. Treatment should be selected and administered as per recommendations for primary uncomplicated UTI or complicated UTI, as appropriate based on a prior history of UTI and the presence of ongoing comorbidities or risk factors. If the patient has not had recurrent infections, the catheter has been removed and no relevant comorbidities are presented as a simple uncomplicated UTI is indicated. Otherwise, patients should be managed as complicated UTIs.

\section{Upper Urinary Tract Infections (Pyelonephritis)}

7.1. Diagnosis. Culture and susceptibility testing should always be performed. Cystocentesis samples should be used for culture whenever possible. If cystocentesis is not possible because of the presence of little urine in the bladder, a sample obtained by urinary catheter is acceptable, but not ideal. Positive cultures should be confirmed by a subsequent cystocentesis sample, whenever possible. Other general principles of diagnosis, as described under Simple Uncomplicated UTI, apply.

Interpretation of susceptibility data should be based on antimicrobial breakpoints for serum rather than urine concentrations. If multiple organisms are isolated, the suspected relative relevance of these should be considered. This assessment would include the bacterial species and colony counts, as is discussed above.

7.2. Treatment. Treatment should be initiated immediately, while awaiting culture and susceptibility results. Initial treatment should involve antimicrobial drugs known to have local or regional efficacy against Gram-negative Enterobacteriaceae, based on the predominance of those organisms in pyelonephritis. If regional data are supportive, treatment with a fluoroquinolone excreted in urine in the active form (e.g., not difloxacin) is a reasonable first choice. If ascending infection is suspected, urine culture results obtained for diagnosis of lower UTI might be the basis of initial therapy. If the upper UTI results from hematogenous spread, initial therapy should be based on cultures of blood or the infected site, whenever available.

While treatment will be started before culture results are available, the culture and susceptibility data should be reviewed when results are received. If combination therapy was initiated and the isolate is susceptible to both drugs, one might be discontinued if supported by evidence of clinical response. If resistance is reported to one of the drugs, that antimicrobial should be discontinued. A second drug to which the isolate is susceptible should be substituted if the patient has not responded sufficiently; substitution is not necessary if patient response has been sufficient. If resistance is reported to both antimicrobials and clinical evidence of improvement is not evident, antimicrobial treatment should be changed to a drug to which the offending organism is susceptible in vitro. Consultation with a specialist is indicated with multidrug-resistant organisms.

Treatment of 4-6 weeks is often recommended. A shorter duration of therapy might be effective; however there is currently inadequate evidence to provide objective recommendations, and 4-6 weeks of treatment is recommended at this time.

7.3. Monitoring Therapy. Urinalysis and culture should be performed 1 week from the start of treatment because of potential severity of disease and long treatment duration. If the same organism is isolated, one should consider adding an antimicrobial to which the organism is susceptible in vitro, if possible. Consultation with a specialist is recommended.

If polymicrobial infection was present initially and only 1 organism remains, consultation with a specialist is recommended to determine the relative clinical relevance of the isolates and treatment plan. Treatment should not necessarily be changed because it is possible that the primary pathogen has been eliminated and changing therapy could result in regrowth of that organism.

Culture is recommended 1 week after cessation of therapy to ensure elimination of infection.

\section{Multidrug-Resistant Infections}

Multidrug-resistant pathogens, including various Enterobacteriaceae, staphylococci, and enterococci, are becoming increasingly problematic. Multidrug-resistant pathogens may be harder to treat because of limited drug choices. There are public health concerns with regard to the potential for zoonotic transmission of resistant pathogens. Additional concerns result from the increasing pressure to use antimicrobials in animals that are critically important in human medicine.

Addressing the globally increasing issue of antimicrobial resistance is complex and difficult. Because of the high incidence of antimicrobial use in UTIs of dogs and cats, veterinarians must be cognizant of the role of inappropriate treatment in the emergence and dissemination of multidrugresistant pathogens. At the same time, the Working Group believes that prudent (and therefore rare) use of certain drugs in the treatment of canine and feline UTIs would constitute a miniscule fraction of overall use of these critical drugs. As such, use of critically important antimicrobials in companion animals can be justified as long as their use is prudent and proper, based on culture and susceptibility data as well as patient care and welfare reasons In particular, the use of drugs such as vancomycin, carbapenems, and linezolid is not justified unless the following criteria are met.

(i) Infection must be documented based on clinical, culture, and cytological abnormalities. The use of 
these drugs for the treatment of subclinical infection is not supported.

(ii) Resistance to all other reasonable options and susceptibility to the chosen antimicrobial must be documented.

(iii) The infection must be potentially treatable. The use of critical drugs in situations where there is little realistic chance of elimination of infection (e.g., failure to remove the underlying cause) is not supported.

(iv) Consultation with someone with expertise in infectious diseases and antimicrobial therapy must be obtained to determine whether there are any other viable options and whether treatment is reasonable.

\section{Conclusions}

Urinary tract disease is a common reason for antimicrobial therapy in dogs and cats. Proper and prompt diagnosis of UTI is required to allow for informed treatment decisions to be made, and careful scrutiny of patient history, clinical signs, urinalysis results, as well as culture and susceptibility data, is required for optimal case management. Successful treatment should not only involve elimination of the clinically apparent infection, it should also do so while minimizing the risks of complications such as struvite urolithiasis, ascending or systemic infection, recurrent infection, or development of antimicrobial resistance. While there are major limitations in available data, including a complete lack of published efficacy studies for dogs and cats, these comprehensive practice guidelines will assist with optimal management of UTIs in dogs and cats.

\section{Acknowledgments}

The guideline development meeting was supported by an unconditional educational grant from Bayer Corporation USA. The authors thank Dr. Jodi Westropp for her review of the guidelines.

\section{References}

[1] L. E. Nicolle, S. Bradley, R. Colgan, J. C. Rice, A. Schaeffer, and T. M. Hooton, "Infectious diseases society of America guidelines for the diagnosis and treatment of asymptomatic bacteriuria in adults," Clinical Infectious Diseases, vol. 40, no. 5, pp. 643-654, 2005.

[2] J. W. Warren, E. Abrutyn, J. Richard Hebel, J. R. Johnson, A. J. Schaeffer, and W. E. Stamm, "Guidelines for antimicrobial treatment of uncomplicated acute bacterial cystitis and acute pyelonephritis in women," Clinical Infectious Diseases, vol. 29, no. 4, pp. 745-758, 1999.

[3] S. Deuster, I. Roten, and S. Muehlebach, "Implementation of treatment guidelines to support judicious use of antibiotic therapy," Journal of Clinical Pharmacy and Therapeutics, vol. 35, no. 1, pp. 71-78, 2010.

[4] T. A. Metjian, P. A. Prasad, A. Kogon, S. E. Coffin, and T. E. Zaoutis, "Evaluation of an antimicrobial stewardship program at a pediatric teaching hospital," Pediatric Infectious Disease Journal, vol. 27, no. 2, pp. 106-111, 2008.
[5] N. R. Toth, R. M. Chambers, and S. L. Davis, "Implementation of a care bundle for antimicrobial stewardship," American Journal of Health-System Pharmacy, vol. 67, no. 9, pp. 746-749, 2010.

[6] J. W. Bartges, "Diagnosis of urinary tract infections," Veterinary Clinics of North America-Small Animal Practice, vol. 34, no. 4, pp. 923-933, 2004.

[7] R. Orenstein and E. S. Wong, "Urinary tract infections in adults," American Family Physician, vol. 59, no. 5, pp. 12251234, 1999.

[8] X. Albert, I. Huertas, I. I. Pereiró, J. Sanfélix, V. Gosalbes, and C. Perrota, "Antibiotics for preventing recurrent urinary tract infection in non-pregnant women," Cochrane Database of Systematic Reviews, no. 3, Article ID CD001209, 2004.

[9] I. S. Gatoria, N. S. Saini, T. S. Rai, and P. N. Dwivedi, "Comparison of three techniques for the diagnosis of urinary tract infections in dogs with urolithiasis," Journal of Small Animal Practice, vol. 47, no. 12, pp. 727-732, 2006.

[10] C. Barbosa-Cesnik, M. B. Brown, M. Buxton, L. Zhang, J. Debusscher, and B. Foxman, "Cranberry juice fails to prevent recurrent urinary tract infection: results from a randomized placebo-controlled trial," Clinical Infectious Diseases, vol. 52, no. 1, pp. 23-30, 2011.

[11] R. G. Jepson and J. C. Craig, "Cranberries for preventing urinary tract infections," Cochrane Database of Systematic Reviews, no. 1, Article ID CD001321, 2008.

[12] E. A. Opperman, "Cranberry is not effective for the prevention or treatment of urinary tract infections in individuals with spinal cord injury," Spinal Cord, vol. 48, no. 6, pp. 451-456, 2010.

[13] D. A. Wing, P. J. Rumney, C. W. Preslicka, and J. H. Chung, "Daily cranberry juice for the prevention of asymptomatic bacteriuria in pregnancy: a randomized, controlled pilot study," Journal of Urology, vol. 180, no. 4, pp. 1367-1372, 2008.

[14] L. J. Bubenik, G. L. Hosgood, D. R. Waldron, and L. A. Snow, "Frequency of urinary tract infection in catheterized dogs and comparison of bacterial culture and susceptibility testing results for catheterized and noncatheterized dogs with urinary tract infections," Journal of the American Veterinary Medical Association, vol. 231, no. 6, pp. 893-899, 2007.

[15] J. Ogeer-Gyles, K. Mathews, J. S. Weese, J. F. Prescott, and P. Boerlin, "Evaluation of catheter-associated urinary tract infections and multi-drug-resistant Escherichia coli isolates from the urine of dogs with indwelling urinary catheters," Journal of the American Veterinary Medical Association, vol. 229, no. 10, pp. 1584-1590, 2006.

[16] S. D. Smarick, S. C. Haskins, J. Aldrich et al., "Incidence of catheter-associated urinary tract infection among dogs in a small animal intensive care unit," Journal of the American Veterinary Medical Association, vol. 224, no. 12, pp. 19361940, 2004. 

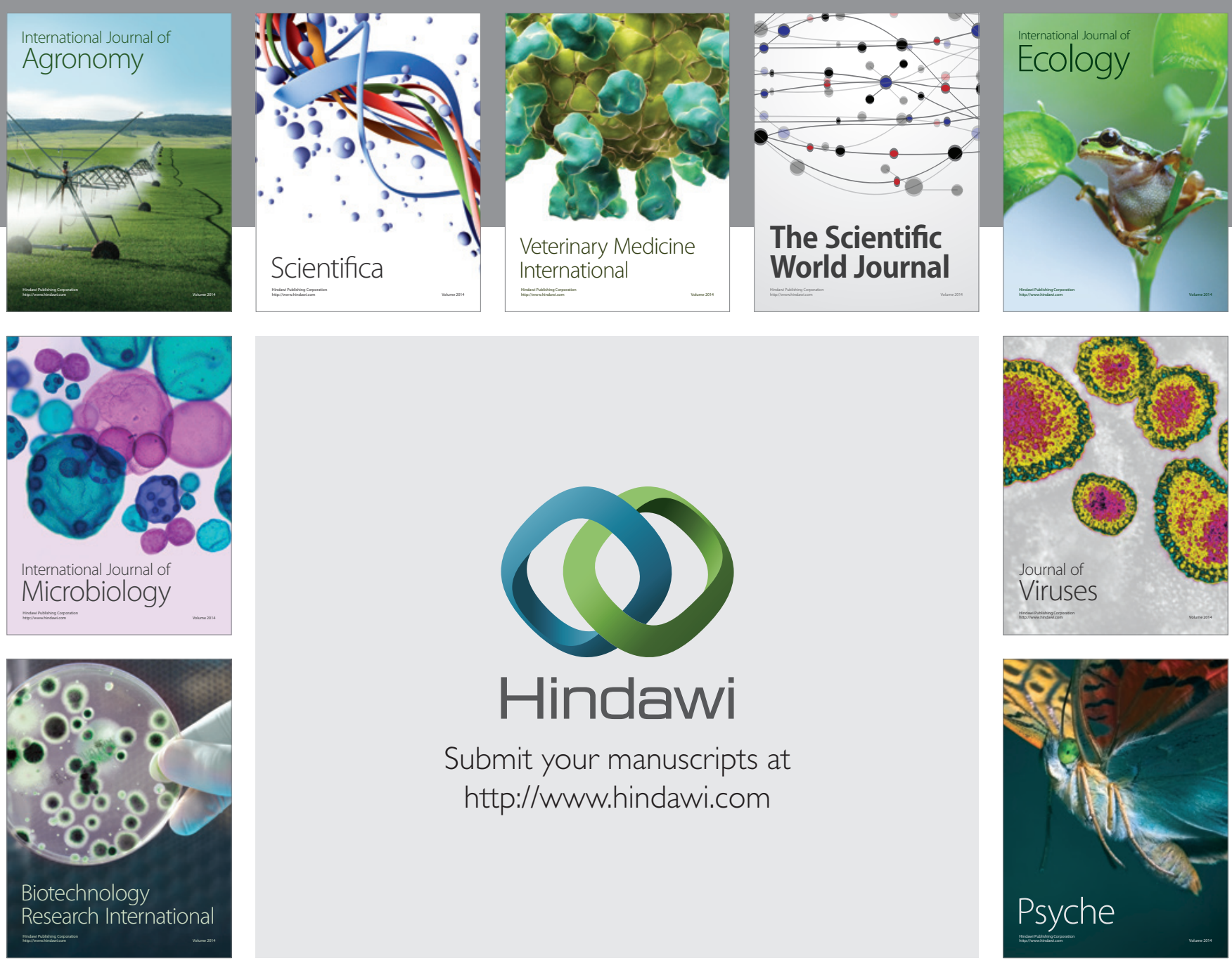

Submit your manuscripts at

http://www.hindawi.com
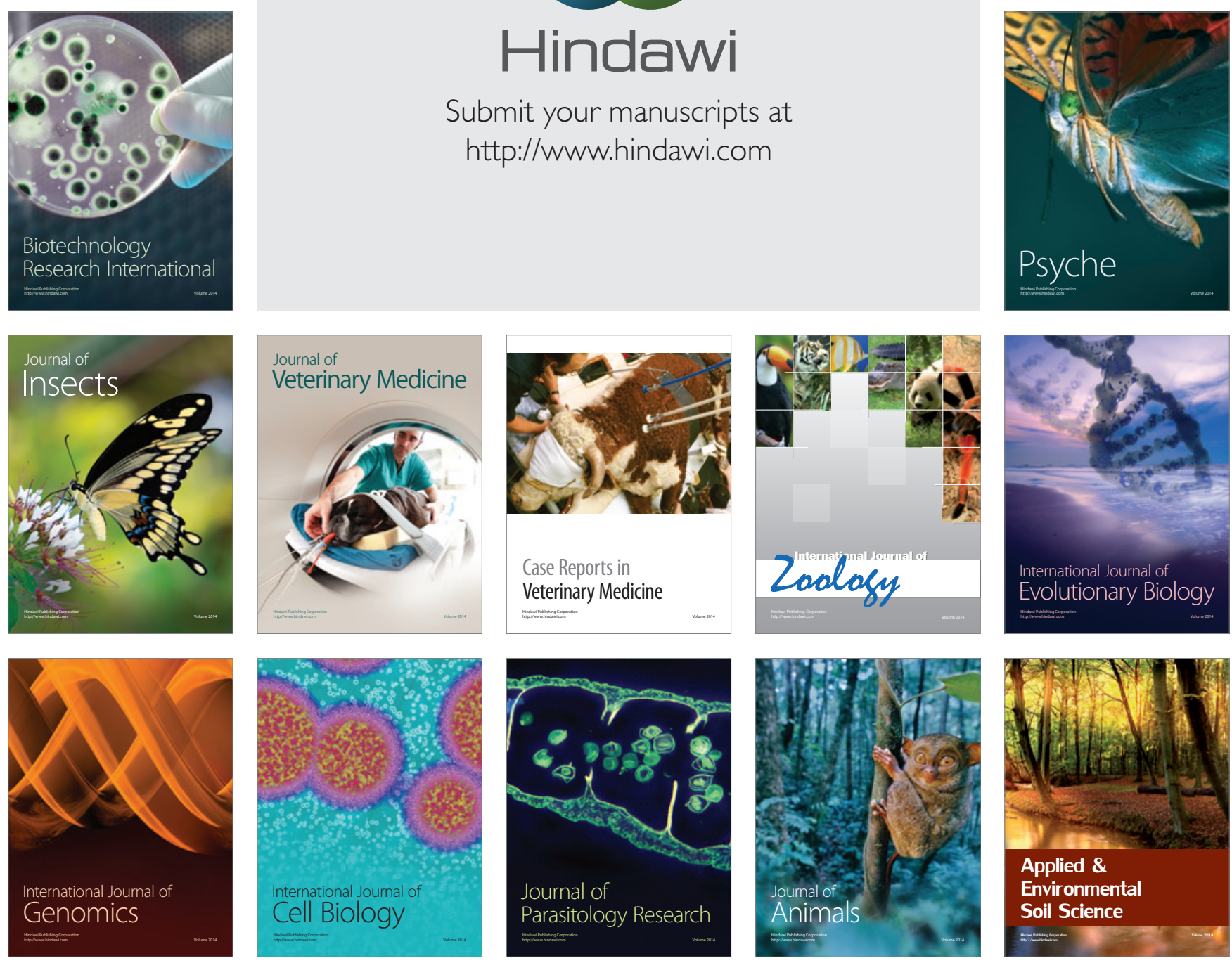